\title{
PATIENTS VIEWS OF NURSE PRESCRIBING: EFFECTS ON CARE, CONCORDANCE AND MEDICINE TAKING
}

Keywords: Dermatology, nurse prescribing, service efficiency, consultation, concordance

\section{Word count: 2960}

Tables: 2

M. Courtenay, Professor of Clinical Practice: Prescribing and Medicines Management, Division of Health and Social Care, University of Surrey

N. Carey, Senior Research Fellow, Division of Health and Social Care, University of Surrey

K. Stenner, Research Fellow, Division of Health and Social Care, University of Surrey

Sandra Lawton, Nurse Consultant Dermatology, Queen's Medical Centre, Nottingham University Hospital NHS Trust

Jill Peters, Dermatology Nurse Practitioner, NHS Suffolk PCT

Molly Courtenay, Professor of Clinical Practice: Prescribing and Medicines

Management, Division of Health and Social Care, University of Surrey

Tel: 01483682511

Email: m.courtenay@surrey.ac.uk

Funding source: Industrial grant

Declaration of competing interest: Nothing to declare

What is known about this topic?

$>$ Nurse prescribers have an important role in the provision of specialist dermatology services

$>$ Flexible consultation times and prescribing knowledge enables nurses to talk to patients about their medicines.

\section{What does this study add?}

$>$ Nurse prescribing can increase the efficiency of dermatology services

$>$ Nurse prescribing supports patient involvement in care which in turn contributes to concordance and adherence to treatment regimes 


\section{Background}

\section{ABSTRACT}

Skin disease can have a huge impact on quality of life for sufferers and their families. Nurses have an important role in the delivery of specialist dermatology services, and prescribing enhances the care they provide. The views of dermatology patients about nurse prescribing are unknown.

\section{Objective}

To explore the views of dermatology patients about nurse prescribing, and its impact on medicines management and concordance.

\section{Patients/methods}

Semi-structured interviews were undertaken with a consecutive sample of 42 patients with acne, psoriasis or eczema who attended the clinics of 7 dermatology specialist nurse prescribers. Primary and secondary care clinics were included to reflect settings in which nurses typically prescribe for patients within specialist dermatology services in England. Interviews addressed the effects of nurse prescribing on care, patient's medicine regime, involvement in treatment decisions and concordance, and influences on medicine taking.

\section{Results}

Patients believed that nurse prescribing improved access to, and efficiency of, dermatology services. Great value was placed on telephone contact with nurses, and local access. Information exchange and involvement in treatment decisions ensured that treatment plans were appropriate and motivated adherence. Nurses' specialist knowledge, interactive and caring consultation style, and continuity of care improved confidence in the nurse and treatment concordance.

\section{Conclusion}

Nurse prescribing can increase the efficiency of dermatology services. Patients experienced active involvement in decisions about their treatment which in turn contributed to concordance and adherence to treatment regimes. This study has important implications for maximising resource use and improving access to, and quality of care, in dermatology specialist services. 


\section{INTRODUCTION}

A lack of trained dermatologists, an increased incidence of skin disease, and an increase in treatment options available for skin conditions has meant greater demand for dermatology services. ${ }^{1}$ It is evident that nurses are essential to dermatology service provision ${ }^{2}$ their roles involving education, facilitating self management, support and advocacy. ${ }^{\mathbf{1}}$ Enhancing the roles of healthcare workers is key to improving accessibility of health care, ${ }^{3}$ and enhancing the roles of specialist nurses to include prescribing has been identified as one way of improving service provision in dermatology. ${ }^{4}$ Over 19,000 nurses ${ }^{5}$ across the United Kingdom (UK) have virtually the same independent prescribing rights as doctors (and the most extended worldwide) and some dermatology specialist nurses have adopted this role in order to support and deliver a range of services to dermatology patients. ${ }^{6}$

In-line with policy literature, ${ }^{7}$ the benefits of nurse prescribing include improved patient access to medicines, better use of health professionals' skills, more flexible working, and improved team working. ${ }^{8}$ Patients are confident in nurse prescribing and this confidence is inspired by nurses' specialist knowledge and a trusting relationship. ${ }^{\mathbf{9}}$ Patients like the continuity of care, ${ }^{\mathbf{1 0 - 1 4}}$ comprehensive information, ${ }^{\mathbf{1 5}} \mathbf{1 1}$, $\mathbf{1 6 , 1 7 , 1 2 , 1 3}$ and holistic care ${ }^{14,17,15}$ that nurses provide.

Despite the huge impact that skin disease can have on quality of life for sufferers and their families, evidence suggests that $40 \%$ do not use their recommended medicines as directed ${ }^{18-21}$. Medication adherence is increased however when patients have a strong relationship with their doctor, feel actively involved in disease management $\mathbf{1 8 - 2 2}$, and receive detailed information and instructions on how to take the medicine $\mathbf{1 8 , 1 9 , 2 1 , 2 2}$. Specialist nurse prescribers report that flexible consultation times and prescribing knowledge better enable them to talk to dermatology patients about their medicines. ${ }^{6}$ This is important as it has been reported ${ }^{2,23}$ that having more time for the consultation and information provision improves clinical outcomes for these patients. However, patients' opinions about the acceptability and impact of nurse prescribing in dermatology are unknown.

\section{PATIENTS AND METHODS}

This qualitative study used semi-structured interviews to explore the views of dermatology patients under the care of a dermatology specialist nurse prescriber. Three or more participants were recruited from the case-load of each of 7 nurses working in specialist dermatology services in primary and secondary care sites across England. The interview schedule was informed by earlier work. ${ }^{6,24,25}$

\section{Recruitment}

Nurses were recruited via the British Dermatological Nursing Group (BDNG) and contacts made through previous work. ${ }^{6}$ Sites reflected the key settings in which nurses typically prescribe for dermatology patients within a specialist dermatology service in primary and or secondary care (see Table 1). Nurses were located across four Strategic Health Authorities including: East Midlands, East of England, South East Coast, and Yorkshire \& Humber. Each had been qualified as a prescriber for more than 2 years. 
Each patient with eczema, psoriasis or acne, who had been prescribed medication by a nurse prescriber when attending a review clinic, was selected to receive a letter from the nurse inviting them to participate in the study, i.e. a consecutive sample $\mathbf{2}^{\mathbf{2 6}}$ of all eligible individuals who attended the clinic. Patients who met the following criteria were excluded and not approached: those who had not received treatment from a nurse prescriber, those who did not have acne, psoriasis or eczema, and patients who did not speak English. The contact details of those willing to participate, were then passed to the researcher and a convenient time to meet was agreed. Participants were invited to take part in an audio-recorded interview lasting between 20-30 minutes.

\section{Data collection}

Interviews were undertaken between March and July 2009. Consent was obtained before each interview. Patients or parents were offered $£ 10$ towards expenses. Interviews were conducted by an experienced qualitative researcher (NC) with a nursing background and in the patients' usual clinic. Parents attended where appropriate. Topics covered by the interview schedule included the effects of nurse prescribing on care, benefits and disadvantages of nurse prescribing, patient's medicine regime, involvement in treatment decisions and concordance, and influences on medicine taking.

\section{Ethics}

Ethical approval for the study was obtained from both University and NHS ethics committees. Research and Development approval was obtained for the study to go ahead in each Hospital and Primary Care Trust.

\section{Analysis}

A thematic analysis, which involved searching across the data set to find repeated patterns of meaning ${ }^{27}$ was conducted on the data. Interviews were coded and categorised with the aid of computer software for qualitative analysis (ATLAS Ti). Through a process of constant comparison, the meaning and relationship between categories were analysed in relation to the research questions in order to produce explanatory themes.

Reliability of coding was enhanced by the independent assessment of transcripts by an additional skilled qualitative researcher. A cross-coding check was completed on a random selection of 10 interviews coded by the second researcher. The second researchers (KS) codes matched those coded by the first researcher (NC) with the exception of two codes which were subsequently included in the analysis following a discussion of differences in code titles.

\section{RESULTS}

Forty two (82.3\%) (of the 51 patients invited to take part in an interview), agreed to participate. A problem with audio-equipment meant that a total of 40 interviews were transcribed and available for analysis (see Table 2 patient profile). 
Five themes were identified. Quotations have been selected to illustrate themes and coded to protect anonymity: $\mathrm{AT}=$ acute trust, $\mathrm{CH}=$ Community Hospital, $\mathrm{CC}=$ Community Clinic, $n=$ nurse number, $\mathrm{p}=$ patient number, $\mathrm{NP}=$ nurse prescriber.

\section{Access to services and efficiency}

All patients were supportive of nurse prescribing. They believed that increasing the numbers of healthcare professionals who could prescribe medicines had increased the number and flexibility of appointments and so access to dermatology services. As fewer people were involved in their care this improved the efficiency with which they were dealt with during their appointments.

"It's just quick and easy. She [NP] can prescribe; she doesn't have to refer me to someone else to get a prescription". AT n1p7

It was said to be easier and faster to gain access to the nurse prescriber than a consultant. Patients $(n=18)$ described how they were able to contact the nurse by telephone between appointments. Access to services in local settings was valued and helped reduce the anxiety associated with attending hospitals. Fast access to a specialist nurse prescriber was valuable and reassuring, especially for patients experiencing flare-ups of their condition and requiring advice at short notice.

"If I've got any problems I don't have to go through the hospital, I can just phone her [NP] straight up and she is at the end of the phone". $\mathrm{CH}$ n2p6

\section{Information giving and continuity of care}

Patients were highly satisfied with the extent of information about treatment benefits, risks and side effects provided by the nurse. Nurses were praised for taking the time to answer questions and checking patient's recall and understanding.

"I have had it [the treatment] really well explained to me at the beginning when I first went on it, then when I came back on to the Y[name of treatment] it was explained to me again." $\mathrm{CH}$ n2p8

Information about side effects meant that patients were less worried when they occurred.

"I started off on a mild dose and it has got gradually higher and higher and I think that has helped the side effects. This month I have gone to my maximum dosage and my skin has gone awful but I know how to deal with the side effects, like all the dry skin.”AT n4p4

Patients were provided with the opportunity to see the same nurse over a period of time. Great importance was placed on this continuity of care as it improved relationships and facilitated discussion of sensitive or embarrassing issues.

"It makes you more open to discuss anything you might be worried about, or any other problems that you have got that might have cropped up." AT n1p3

As the nurse knew the patient and their treatment regime, more time could be spent discussing treatments and medicines regimes, thus providing patients with the 
opportunity to increase their understanding.

"You build up a relationship, you know who to expect, you know what they are going to be like and they always seems to have time to listen to me. With the nurse I have been able to sit and have a chat, learn more and understand the tablets." AT n7p2

\section{Confidence and concordance}

Patients were unanimously confident in the ability of the nurse prescriber. Confidence was inspired by the nurses' level of specialist knowledge, their experience with specific treatment regimes, and that nurses knew their own limitations and would refer to the doctor for further advice.

"If she has any queries she will certainly see X [dermatology consultant], and if she queries anything she phones through to my doctor [GP] regularly to check up that I am taking it" $\mathrm{CH}$ n2p2

Confidence in the decisions made by the nurse was further increased when the effects of treatment were noticeable to patients.

"Once you can see some improvement you feel confident that you have done the right thing, you are confident in X $[\mathrm{NP}]$ " CC n3p3

All participants reported that they were actively involved in decision-making about their treatment. Concordance occurred where patients' views were incorporated into treatment plans and this boosted patients' confidence in the nurse's ability as a prescriber.

"We have reduced it [the treatment] and now we are going to try and reduce it a little bit and see how it goes. Little bit anxious because every time I have reduced it before it has got worse, but I discussed that with the nurse, but if it started to flare up again would I be able to go back and she reassured me that I would." $\mathrm{CH}$ n2p8

Written treatment plans were frequently used to reinforce the information provided. While many patients described problems they had encountered in the past, the majority said that did not have any problems adhering to their current regimen. Having a regimen that was simple and easy, as well as feeling happy with the treatment decision helped patients adhere to their regimen.

"She [NP] has always done a written plan. She always puts down how many days to use certain creams and wet wrap bandages. That's very useful." AT n5p2

\section{Differences between doctors and nurses}

Most patients were equally satisfied with care provided by the consultant dermatologist compared with the nurse prescriber, however differences in opinions were expressed with regards to the level of knowledge between specialist nurses and different types of doctors. Due to their extensive training, doctors were thought to have greater knowledge overall, and one group of patients felt that doctors, particularly specialist consultants, were more knowledgeable in this area. By contrast, 
others patients believed dermatology nurses offered a superior service, especially compared to GPs, as they had a high level of expertise in the area of dermatology.

"She $[\mathrm{NP}]$ is more professional in dermatology than the GP is. The GP knows everything, obviously they have to. They are general aren't they, but she is probably more professional in what she is doing". $\mathrm{CH} n 2 \mathrm{p} 7$

Other key differences described by patients were that nurses appeared to have more time to spend with them, were more approachable, easier to talk to and made them feel more relaxed. Nurse consultations frequently involved a broader discussion about lifestyle behaviours; an approach valued by parents as the nurse was able to distinguish between behaviour potentially related to the skin condition and normal childhood development. Nurses were reported to be highly attentive, understanding and demonstrated good listening skills. As a result, patients gained more information and understanding, felt less rushed, more cared for, and more satisfied.

"Doctors don't seem to have time to explain everything where X [NP] does..... when you are asking a doctor you feel they are answering because they just have to answer" AT n1 p3.

Nurses were also said to show a greater level of concern and interest in patient's skin condition and general well being. An exception to this was expressed by one parent who was unable to resolve concerns and frustrations over her child's ongoing skin condition. Sharing concern was particularly important to patients many of whom had suffered psychological distress as a result of their skin condition.

"I think it is like more attention to me and my problems rather than the problem itself. She [NP] remembers things that have happened in the past and she will ask me about those things. Even if they are not written down she will have remembered them rather than just following on from the notes". AT n4p4

\section{Patient concerns}

Few patients had concerns about nurse prescribing in this setting. Patients were reassured by the fact they were dealing with a specialist nurse who was responsible for managing a very specific condition and treatment regimen. One patient felt confident so long as the nurse was merely continuing the treatment rather than deciding upon it, and two lacked confidence that a nurse would know what to do if something 'serious' was wrong. Several patients commented on the need to assume that nurses had adequate training to undertake the prescribing role.

"Obviously if a person is given permission to write prescriptions they are obviously well qualified and I'm quite happy with that" CC n3p2

\section{DISCUSSION}

This is the first study to explore the views of dermatology patients' about nurse prescribing and has important implications for service delivery.

Patients reported that nurse prescribing improved access to services by increasing the number of available appointments, offering telephone access, providing local services, and improving efficiency. These are all aspects of services that have previously highlighted as important to the care for patients with chronic skin conditions ${ }^{6,28,4}$, and 
align with previous research on the views of healthcare professionals working in dermatology services ${ }^{6}$ and patients views about the efficiency of nurse prescribing ${ }^{9,29}$

Our findings indicate that the care provided by nurses in this setting is in line with guidance that calls for a greater alignment between the views of the prescriber and patient if medication adherence is to be improved and concordance successfully achieved. ${ }^{18,19,21}$ Patients reported to be involved in treatment decisions and that their views were considered during care planning. They were happy with the amount and type of information given to them and the consistency with which it was provided. This is in contrast to earlier research in which inconsistencies in the information patients receive about their medicines from nurse prescribers have been reported. ${ }^{6,16}$. The findings therefore add to the evidence that dermatology nurse specialists' demonstrate good practice as set out in guidance on their role ${ }^{1}$ key elements of which are to provide adequate information and emotional support to patients and their families. Compared to doctors, nurses were said to be more concerned about patients well being, and easier to talk to. They were also said to make patients feel more relaxed which made them less concerned about consultation time. This is consistent with the views of healthcare professionals, ${ }^{11}$ where nurses are reported to be more approachable than doctors, to have better listening skills, to adopt a more supportive role, and to have greater flexibility with regards to consultation times enabling the encouragement of adherence to treatment regimes in chronic skin conditions. This is an important finding given that $40 \%$ of dermatology patients do not use their medicines as directed. ${ }^{18-21}$

As in previous research, ${ }^{9}$ participants highly rated the consultation style and continuity of care they had with the specialist nurse. In addition, our findings illustrate how continuity of care enabled more efficient use of consultation time. The importance to patients of having a personal and long-term relationship with their healthcare provider, particularly when experiencing a psychological or significant health problem, such as a chronic skin complaint, has previously been reported. ${ }^{30}$ Nurse prescribers themselves are reported to value continuity as it enhances their ability to evaluate the outcomes of prescribing decisions and learn about dermatology treatments. ${ }^{6}$

Patients reported that a high level of trust and confidence in the nurse prescriber, as has been identified previously. ${ }^{31}$ Our findings suggest this stemmed from a number of inter-related factors including nurses' level of specialist knowledge, patient involvement in decisions about their treatment, nurses' thoroughness during the consultation, the effects of treatment being noticeable to patients, and the long-term relationships nurses had with patients. This is important as strength of relationship and trust in medical prescribers has been shown to improve medication adherence $18,19,22,32$

Nurses were said to be very knowledgeable about the treatments they provided and, as previously reported, ${ }^{9}$ patients were reassured by nurses specialist knowledge and experience. Although equally satisfied with the care provided by specialist doctors and nurses, specialist nurses were seen as providing a more superior service than GPs. Specialist knowledge and its effects on quality of care has been reported previously ${ }^{33}$ 
Our work could have been strengthened if a greater number of patients working in community clinics had been recruited. In addition, nurses may have volunteered because of their high level of competence and experience in dermatology; therefore findings, which were highly positive, may not represent the experiences of all patients attending a specialist dermatology service. However, nurses selected do reflect the key settings in which specialist nurses typically prescribe for dermatology patients.

Despite these limitations, our findings demonstrate how nurse prescribing can increase the efficiency of dermatology services. They also demonstrate how nurse prescribing can support patient involvement in care which in turn contributes to concordance and adherence to treatment regimes. Further research is required to explore this contribution. At a time of ongoing financial restraints within the NHS this study has important implications for maximising resource use and improving access to and quality of care in dermatology specialist services.

\section{Acknowledgements}

This work was funded by an unrestricted research grant from Galderma UK. We would like to thank the BDNG for their advice and support and all those who participated in this research. 


\section{REFERENCES}

1) Dermatology Workforce Group (DWG) Models of integrated service delivery in dermatology. London: DWG 2007

2) Courtenay, M \& Carey, N Nurse-led care in dermatology: a review of the literature. British Journal of Dermatolgy 2006,. 154, pp1-6.

3) World Health Organisation (WHO). The World Health Report. Working Together for Health. Geneva: WHO 2006

4) DoH. Shifting Care Closer to Home. London: DoH 2007.

5) Cully, F. Professional considerations for nurse prescribers. Nursing Standard, 24 (43), 55-60

6) Courtenay, M \& Carey, N, Stenner, K. Nurse prescriber-patient consultations: A case study in dermatology. Journal of Advanced Nursing 2009; 65 (6), 1207-1217.

7) DoH. Improving patient's access to medicines: A guide to implementing nurse and pharmacist independent prescribing within the NHS in England. London: DoH 2006

8) Bradley E. \& Nolan P. Impact of nurse prescribing: a qualitative study. Journal of Advanced Nursing 2007; 59 (2), 120-128

9) Courtenay, M., Stenner, K., Carey, N. The views of patients with diabetes about nurse prescribing. Diabetes Medicine 2010 10.1111/j.1464-5491.2010.03051

10) Wix, S. Independent nurse prescribing in the mental health setting. Nursing Times 2007; 103 (44), 30-31

11) Latter, S., Maben, J, Myall, M., Courtenay, M., Young, A., Dunn, N. An evaluation of extended formulary independent nurse prescribing. Executive summary of final report. London: DoH 2005.

12) Brooks N, Otway C, Rashid C, Kilty E, Maggs C. The patients' view; the benefits and limitations of nurse prescribing. British Journal of Community Nursing 2001; 6: 342-348

13) Luker, K.A., Austin, L., Hogg, C., Ferguson, B., Smith, K. Nurse-patient relationships: the context of nurse prescribing. Journal of Advanced Nursing 1998; 28 (2) $235-242$.

14) Harrison, A. Mental health service users' views of nurse prescribing. Nurse Prescribing 2003; 1 (2), 78-85.

15) Page, D., Grant, G., Maybury, C. Introducing nurse prescribing in a memory clinic. Dementia 2008; 7 (1), 139-160 
16) Latter, S., Maben, J., Myall, M., Young, A. Perceptions and practice of concordance in nurses' prescribing consultations: Findings from a national questionnaire survey and case studies of practice in England. International Journal of Nursing Studies 2007; 44, 9-18.

17) Jones, M., Bennett, J., Lucas, B., Miller, D., Gray, R. Mental health nurse supplementary prescribing: Experiences of mental helath nurses, psychiatrists and patients. Journal of Advanced Nursing 2007; 59 (5), 488-496.

18) Richards HL, Fortune DG, Griffiths CEM. Adherence to treatment in patients with psoriasis. Journal of the European Academy of Dermatology and Venereology. 2006;20:370-9.

19) Feldman SR, Horn EJ, Balkrishnan R, Basra MK, Finlay AY, McCoy D, et al. Psoriasis: Improving adherence to topical therapy. Journal of the American Academy of Dermatology. 2008;59:1009-16.

20) Hugtenburg JG, Blom ATG, Kisoensingh SU. Inital Phase of chronic medication use;patients reasons for discontinuation. British Journal of Pharmacology. 2005;61(3):352-4.

21) Nunes V, Neilson J, O'Flynn N, Calvert N, Kuntze S, Smithson H, et al. Clinical Guidelines and Evidence Review for Medicines Adherence: involving patients in decisions about prescribed medicines and supporting adherence. London: National Collaborating Centre for Primary Care and Royal College of General Practitioners2009.

22) Storm A, Benfeldt E, Andersen SE, Serup J. A prospective study of patient adherence to topical treatments: $95 \%$ of patients underdose. Journal of the American Academy of Dermatology. 2008;59:975-80.

23) Moore, E.J., Williams, A., Manias, E., Varigos, G., Donath, S. Australian Journal of Dermatology 2009; 50, 100-106

24) Courtenay, M Carey, N., Burke, J. Independent extended and supplementary prescribing practice: A national questionnaire survey. International Journal of Nursing Studies. 2007, 44 (7), 1093-1101

25) Carey, N. \& Courtenay, M. Supplementary nurse prescribing for patients with skin conditions: a national questionnaire survey. Journal of Clinical Nursing. 2007, $16,1230-1237$

26) Lunsford, T.R, Lunsford, B.R . RESEARCH FORUM--The Research Sample, Part I: Sampling. Journal of Prosthetics and Orthotics Online Library 1995, 7 (3) 15. Accessed 20 $0^{\text {th }}$ September 2010 http://www.oandp.org/jpo/library/1995_03_105.asp

27) Braun V. \& Clark V. Using thematic analysis in psychology. Qualitative Research in Psychology 2006, 3, 77-101.26 
28) DoH. Modernisation Agency NHS. Action on Dermatology Good Practice Guide. London: DoH 2003

29) Drennan, J., Naughton, C., Allen, D., Hyde, A., Felle, P., O’Boyle, K., Yreacy, P., Butler, M (2009). National Independent Evaluation of the Nurse and Midwife Prescribing Initiative. Dublin: University College Dublin 2009

30) Kearley KE, Freeman GK, Heath A. An exploration of the value of the personal doctor-patient relationship in general practice. British Journal of General Practice. 2001;51:712-8.

31) Berry, D, Courtenay, M, Bersellini, E. Attitudes towards, and information needs in relation to supplementary nurse prescribing in the UK: an empirical study. Journal of Clinical Nursing 2006; 15, 22-28.

32) Brown KK, Rehmus WE, Kimball AB. Determining the relative importance of patient motivations for non-adherence to topical corticosteroid therapy in psoriasis. Journal of the American Academy of Dermatology. 2006;55:607-13.

33) Stenner, K. \& Courtenay, M. The benefits of nurse prescribing according to nurses prescribing for patients in pain. Journal of Advanced Nursing 2008, 63 (1), 27 35 
Table 1: Nurse Prescriber Site Information

\begin{tabular}{|c|l|l|l|c|c|}
\hline Site & \multicolumn{1}{|c|}{ Location } & \multicolumn{1}{c|}{ Setting } & \multicolumn{1}{c|}{ Trust } & $\begin{array}{c}\text { Type of } \\
\text { prescribing }\end{array}$ & $\begin{array}{c}\text { No of patient } \\
\text { interviews }\end{array}$ \\
\hline 1 & Yorkshire & Secondary care- OP & Acute trust & IP & 7 \\
\hline 2 & South East Coast & Secondary care- OP & Community Hospital & IP & 8 \\
\hline 3 & East of England & Primary care & Community Clinic & IP & 5 \\
\hline 4 & East Midland & Secondary care- OP & Acute trust & SP & 7 \\
\hline 5 & East Midlands & Secondary care- OP & Acute trust & IP & 7 \\
\hline 6 & East of England & Primary care & Community Hospital & IP & 3 \\
\hline 7 & South East Coast & Secondary care- OP & Acute trust & IP & 5 \\
\hline
\end{tabular}

OP-out-patients IP: nurse independent prescribing SP: nurse supplementary prescribing

Table 2: Participant information

\begin{tabular}{|c|c|c|}
\hline Age & Range & Mean \\
\hline & $<1 \mathrm{yr}-81$ years & 32.4years \\
\hline $\begin{array}{l}<16 \text { yrs } n=7,16-25 \text { yrs } n=16 \\
26-45 y \text { yrs } n=5,45-65 \text { yrs } n=5 \\
>65 y r s n=8\end{array}$ & $\begin{array}{c}\text { Median } \\
19.5 \text { years }\end{array}$ & \\
\hline Gender & & $\%$ \\
\hline Male & $\mathrm{n}=21$ & $50 \%$ \\
\hline Female & $\mathrm{n}=21$ & $50 \%$ \\
\hline Ethnicity & & $\%$ \\
\hline White & $\mathrm{n}=36$ & $85.7 \%$ \\
\hline Other ethnic background & $\mathrm{n}=6$ & $14.3 \%$ \\
\hline Skin condition & & $\%$ \\
\hline Eczema & $\mathrm{n}=14$ & $33.4 \%$ \\
\hline Acne & $\mathrm{n}=19$ & $45.2 \%$ \\
\hline Psoriasis & $\mathrm{n}=9$ & $21.4 \%$ \\
\hline Current Treatment & & $\%$ \\
\hline Topical treatment only & $\mathrm{n}=14$ & $33.3 \%$ \\
\hline Systemic & $n=23$ & $54.8 \%$ \\
\hline Topical \& Systemic & $\mathrm{n}=5$ & $11.9 \%$ \\
\hline Number of visits with nurse & & $\%$ \\
\hline First visit & $\mathrm{n}=2$ & $4.8 \%$ \\
\hline 2-12 visits over the last year & $\mathrm{n}=25$ & $59.5 \%$ \\
\hline Multiple visits $1-3$ years & $\mathrm{n}=12$ & $28.6 \%$ \\
\hline Multiple visits 3-13 years & $n=3$ & $7.1 \%$ \\
\hline
\end{tabular}


